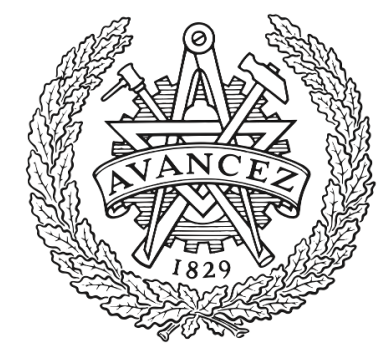

\title{
CHALMERS
}

UNIVERSITY OF TECHNOLOGY

\section{Tracking Position and Orientation Through Millimeter Wave Lens MIMO in 5G Systems}

Downloaded from: https://research.chalmers.se, 2023-04-26 01:19 UTC

Citation for the original published paper (version of record):

Shahmansoori, A., Uguen, B., Destino, G. et al (2019). Tracking Position and Orientation Through Millimeter Wave Lens MIMO in 5G Systems. IEEE Signal Processing Letters, 26(8): 1222-1226. http://dx.doi.org/10.1109/LSP.2019.2925969

N.B. When citing this work, cite the original published paper.

(O2019 IEEE. Personal use of this material is permitted.

However, permission to reprint/republish this material for advertising or promotional purposes 


\title{
Tracking Position and Orientation through Millimeter Wave Lens MIMO in 5G Systems
}

\author{
Arash Shahmansoori, Bernard Uguen, Member, IEEE, Giuseppe Destino, Member, IEEE, Gonzalo \\ Seco-Granados, Senior Member, IEEE, and Henk Wymeersch, Member, IEEE
}

\begin{abstract}
Millimeter wave signals and large antenna arrays are considered enabling technologies for future 5G networks. Despite their benefits for achieving high data rate communications, their potential advantages for tracking of the location and rotation angle of the user terminals are not well investigated. A joint heuristic beam selection and user position and orientation tracking approach is proposed. First, the user location is tracked in the uplink by joint beam selection together with time-of-arrival (TOA) and angle-of-arrival (AOA) tracking at the base station (BS). Then, the user rotation angle is obtained using the location information by joint beam selection and tracking at the mobile station (MS). The beam selection, TOA and AOA tracking at the BS and MS are performed during the data transmission phase. Numerical results demonstrate that the proposed method performs close to the estimated position and rotation angle in the training phase with reduced complexity and reduced number of required pilots for the estimation.
\end{abstract}

Index Terms-5G networks, millimeter wave, lens arrays, position and orientation tracking, heuristic beam selection.

\section{INTRODUCTION}

$\mathbf{M}$ ILLIMETER WAVE and massive multiple-inputmultiple-output (MIMO) will likely be adopted technologies in fifth generation (5G) communication networks, thanks to a number of favorable properties. Particularly, by exploiting the carrier frequencies beyond $30 \mathrm{GHz}$ and large available bandwidth, millimeter wave ( $\mathrm{mm}$-wave) can provide high data rate. This can be obtained through dense spatial multiplexing with large antennas [1], [2]. Despite the aforementioned properties that are desirable for $5 \mathrm{G}$ services, there are a number of challenges regarding mm-wave communications. One of the most important challenges is the severe path loss at high carrier frequencies. The loss in signal-to-noise ratio (SNR) is compensated through beamforming at the transmitter and/or receiver resulting in highly directional links [3]-[5].

Arash Shahmansoori and Bernard Uguen are with the Institute of Electronics and Telecommunications of Rennes, Université de Rennes 1,35042 Rennes, France, emails: arash.mansoori65@gmail.com and Bernard.Uguen@univ-rennes1.fr. Gonzalo Seco-Granados is with the Department of Telecommunications and Systems Engineering, Universitat Autònoma de Barcelona, 08193 Barcelona, Spain, email: gonzalo.seco@uab.cat. Henk Wymeersch is with the Department of Electrical Engineering, Chalmers University of Technology, 41296 Gothenburg, Sweden, email: henkw@chalmers.se. Giuseppe Destino is with the center for wireless communications, University of Oulu, 90014 Oulu, Finland, and visiting research fellow at King's College London, email: giuseppe.destino@ee.oulu.fi. This work was financially supported by M5HESTIA (mmW Multi-user Massive MIMO Hybrid Equipment for Sounding, Transmissions and HW Implementation) project, the VINNOVA COPPLAR project, funded under Strategic Vehicle Research and Innovation grant nr. 2015-04849, FALCON (Fundamental of simultaneous localization and communications) funded by the Academy of Finland, and R\&D Projects of Spanish Ministry of Economy and Competitiveness TEC2017-89925-R. (Corresponding author: Arash Shahmansoori.)
A possible way for low-cost implementation of mm-wave MIMO is achieved by using switching circuits together with lens antenna arrays [6]-[12]. Position-based beamformer design requires the knowledge of propagation channel, e.g., user position, scatterer locations, and so on. The relative location of transmitter and receiver can be obtained using the estimated AOA/angle-of-departure (AOD) for the line-of-sight (LOS) condition [13]. To this end, position and orientation estimation was previously explored in [14]-[19] and in [17], [20], [21] for mm-wave and massive MIMO systems for static channels. To speed up initial access between nodes, a location-aided beamforming method was proposed in [22].

In the case of dynamic channels, a beam switching approach was suggested for tracking of AOA in [14]. A link by link mm-wave AOA/AOD and channel gain tracking was proposed in [23], while a tracking solution for all the links was investigated in [24]. In [25] and [26] different solutions were proposed mainly based on AOA tracking for mm-wave and Terahertz lens antenna arrays by Markov model and a temporal variation law of the physical direction, respectively. All of the aforementioned papers propose the solutions for static channels and AOA based tracking for time-varying channels. However, for tracking the user location and orientation it is essential to consider the combination of TOA and AOA.

In this letter, we propose a joint beam selection position and orientation tracking method using a lens antenna array with one BS. The proposed method tracks the channel parameters with a heuristic beam selection method based on the angular uncertainties provided by the an extended Kalman filter (EKF) in the uplink followed by a downlink transmission. This enables tracking of the position and rotation angle of the user with reduced number of required beams within the observation time. From the simulation results, it is observed that the proposed algorithm provides position and rotation angle estimates during the data transmission phase with similar accuracy to those obtained during the training phase while involving a reduced complexity and number of pilot transmissions.

\section{Proposed Method}

In this section, a joint heuristic beam selection and tracking method is proposed for a mm-wave MIMO system with a lens antenna array. The mm-wave lens MIMO channel model in the uplink (UL) for the $n$-th subcarrier is obtained as [10], [27]

$$
\check{\mathbf{H}}_{\mathrm{UL}}[n]=\sum_{k=0}^{K} \gamma_{n}\left(\tilde{h}_{k}, \tau_{k}\right) \boldsymbol{\chi}_{\mathrm{BS}, k} \boldsymbol{\chi}_{\mathrm{MS}, k}^{\mathrm{T}},
$$


where $\gamma_{n}\left(\tilde{h}_{k}, \tau_{k}\right)$ is defined as $\gamma_{n}\left(\tilde{h}_{k}, \tau_{k}\right)=$ $\tilde{h}_{k} e^{-j 2 \pi n \tau_{k} /\left(N T_{s}\right)}$ in which $\tau_{k}$ is the delay of the $k$ th path, $K+1$ denotes the total number of paths with the LOS indexed by the subscript zero, $N$ is the number of subcarriers, $T_{s}=1 / B$ is the sampling period, and $\tilde{h}_{k}=\sqrt{\left(N_{\mathrm{BS}} N_{\mathrm{MS}}\right) / \rho_{k}} h_{k}$ in which $\rho_{k}$ denotes the path loss with complex channel gain of $h_{k}$. The term $\chi_{\mathrm{MS}, k}$ is an $N_{\mathrm{MS}} \times 1$ vector denoting lens array with $N_{\mathrm{MS}}$ antenna elements and the entries $\chi_{\mathrm{MS}}\left(\frac{d}{\lambda_{c}} \sin \left(\phi_{k}\right)-\frac{i}{N_{\mathrm{MS}}}\right)$ with $\phi_{k}$ being the AOA in the downlink (DL) for $-\left(N_{\mathrm{MS}}-1\right) / 2 \leq i \leq\left(N_{\mathrm{MS}}-1\right) / 2$ where $\chi_{\mathrm{MS}}(\phi)=\sin \left(\pi N_{\mathrm{MS}} \phi\right) /\left(\sqrt{N_{\mathrm{MS}}} \sin (\pi \phi)\right)$, and $\chi_{\mathrm{BS}, k}$ denotes an $N_{\mathrm{BS}} \times 1$ lens array with $N_{\mathrm{BS}}$ antenna elements and defined similarly by replacing the subscript MS by BS and the downlink AOA (DL-AOA) $\phi_{k}$ by AOA in the UL $\theta_{k}$. Using the information provided by the LOS path, the goal is to track the user position ${ }^{1} \mathbf{p}=\mathbf{q}+c \tau_{0}\left[\cos \left(\theta_{0}\right), \sin \left(\theta_{0}\right)\right]^{\mathrm{T}}$ where $\mathbf{q}$ denotes the location of the BS assumed to be known and $c$ is the speed of light, and rotation angle $\alpha=\pi+\theta_{0}-\phi_{0}$ with reduced number of pilot transmissions. It is assumed that the BS does not move and tracks the location of the MS, and the MS tracks its rotation angle using the location information provided by the BS.

\section{A. Measurement and State Equations}

A continuous white noise acceleration (CWNA) model defines the state evolution used for tracking DL-AOA/uplink AOA (UL-AOA), and TOA [28]. The state vector for the LOS path can be written as

$$
\boldsymbol{\psi}_{0}^{[m]}=\left[\begin{array}{ll}
\left(\boldsymbol{\eta}_{0}^{[m]}\right)^{\mathrm{T}} & \left(\dot{\boldsymbol{\eta}}_{0}^{[m]}\right)^{\mathrm{T}}
\end{array}\right]^{\mathrm{T}},
$$

where $\boldsymbol{\eta}_{0}^{[m]}=\left[\tau_{0}^{[m]}, \theta_{0}^{[m]}, \phi_{0}^{[m]}\right]^{\mathrm{T}}$ and $\dot{\boldsymbol{\eta}}_{0}^{[m]}=$ $\left[\dot{\tau}_{0}^{[m]}, \dot{\theta}_{0}^{[m]}, \dot{\phi}_{0}^{[m]}\right]^{\mathrm{T}}$. The terms $\theta_{0}^{[m]}$ and $\phi_{0}^{[m]}$ denote the UL-AOA and DL-AOA for the LOS path at the time instant $m$, respectively. Similarly, $\tau_{0}^{[m]}$ denotes the TOA for the LOS path. Finally, the parameters $\dot{\tau}_{0}^{[m]}, \dot{\theta}_{0}^{[m]}$, and $\dot{\phi}_{0}^{[m]}$ denote the rate-of-change of the TOA, UL-AOA, and DL-AOA for the block duration $T_{B}$, the time between two instants $m$ and $m+1$, respectively. Assuming CWNA model, the state evolution model can be written as

$$
\boldsymbol{\psi}_{0}^{[m]}=\boldsymbol{\Phi} \boldsymbol{\psi}_{0}^{[m-1]}+\mathbf{u}_{0}^{[m]},
$$

where $\mathbf{u}_{0}^{[m]}$ denotes the state noise with $\mathbb{E}\left[\mathbf{u}_{0}^{[m]}\left(\mathbf{u}_{0}^{[m]}\right)^{\mathrm{T}}\right]=$ $\mathbf{Q}_{0}^{[m]}$. In general, the bi-azimuth generalized Von-Mises-Fisher (VMF) distribution for joint DL-AOA/UL-AOA or its approximation by a 2-D truncated Gaussian pdf can be applied for directional data [29], [30]. In this case, we apply the approximation with a 2-D truncated Gaussian pdf with $\sigma_{\phi_{0}}$, $\sigma_{\theta_{0}}$, and $\rho_{\theta \phi, 0}$ denoting the direction spreads of the AOA, AOD, and cross correlation for the LOS path, respectively. Moreover, the amount of noise would depend on $T_{B}$. The state transition matrix $\Phi \in \mathbb{R}^{6 \times 6}$ is defined as

$$
\boldsymbol{\Phi}=\left[\begin{array}{cc}
\mathbf{I}_{3} & T_{B} \mathbf{I}_{3} \\
\mathbf{0}_{3} & \mathbf{I}_{3}
\end{array}\right]
$$

${ }^{1}$ The extension to the multi-user scenario is an exciting topic for future research.
For $m=1$, the entries of $\boldsymbol{\psi}_{0}^{[m-1]}$ in (3) are initialized as: $\tau_{0}^{[0]}=\hat{\tau}_{0}, \phi_{0}^{[0]}=\hat{\phi}_{0}$, and $\theta_{0}^{[0]}=\hat{\theta}_{0}$ where $\hat{\tau}_{0}, \hat{\phi}_{0}$, and $\hat{\theta}_{0}$ are obtained from the training phase. The rate-ofchange terms ${ }^{2}$ are initialized by two consecutive estimates of $\boldsymbol{\eta}_{0}[m]=\left[\tau_{0}^{[m]}, \theta_{0}^{[m]}, \phi_{0}^{[m]}\right]^{\mathrm{T}}$ as: $\dot{\tau}_{0}^{[1]}=\left(\tau_{0}^{[1]}-\tau_{0}^{[0]}\right) / T_{B}$, $\dot{\theta}_{0}^{[1]}=\left(\theta_{0}^{[1]}-\theta_{0}^{[0]}\right) / T_{B}$, and $\dot{\phi}_{0}^{[1]}=\left(\phi_{0}^{[1]}-\phi_{0}^{[0]}\right) / T_{B}$.

For tracking of the channel parameters, the EKF is applied with the state comprising the LOS delay, DL-AOA, UL-AOA, and their corresponding rates of changes, with the linear process model and nonlinear measurement equations in the downlink and the uplink. These parameters are initially available at the BS and the MS from the training/initial access phase [19]. The measurement equation in the uplink is obtained for the orthogonal frequency division multiplexing (OFDM) transmission as

$$
\check{\mathbf{y}}_{r}^{\mathrm{ul},[m]}=\mathbf{z}_{0}^{\mathrm{ul}}\left(\boldsymbol{\eta}_{\mathrm{ul}, 0}^{[m]} ; \phi_{0}^{[m]}\right)+\sum_{l=1}^{K} \mathbf{z}_{0}^{\mathrm{ul}}\left(\boldsymbol{\eta}_{\mathrm{ul}, l}^{[m]} ; \phi_{l}^{[m]}\right)+\check{\mathbf{n}}^{[m]},
$$

where $\boldsymbol{\eta}_{\mathrm{ul}, k}^{[m]}=\left[\tau_{k}^{[m]}, \theta_{k}^{[m]}\right]^{\mathrm{T}}$, and $\check{\mathbf{y}}_{r}^{\mathrm{ul},[m]}$ denotes the received signal vector of size $N M_{\mathrm{BS}} \times 1$ which $M_{\mathrm{BS}}$ is the number of received beams at the BS. In (5), the first term denotes the received signal from the LOS, and the second term denotes the superposition of all the other $K$ non-line-of-sight (NLOS) paths acting as an added ${ }^{3}$ term to the Gaussian measurement noise vector $\check{\mathbf{n}}^{[m]} \in \mathbb{C}^{N M_{\mathrm{BS}}}$ with zero mean and variance $N_{0} / 2$ per real dimension.

The $^{4}$ TOA-AOA are the parameters to be tracked for the block index $m$ in the BS, and $\mathbf{z}_{0}^{\mathrm{ul}}\left(\boldsymbol{\eta}_{\mathrm{ul}, k}^{[m]} ; \phi_{k}^{[m]}\right)$ denotes

$$
\begin{aligned}
\mathbf{z}_{0}^{\mathrm{ul}}\left(\boldsymbol{\eta}_{\mathrm{ul}, k}^{[m]} ; \phi_{k}^{[m]}\right)=\tilde{h}_{k}^{[m]}\left(\mathbf{X}_{0}^{\mathrm{T}}\left(\mathbf{F}_{\mathrm{MS}, 0}^{[m-1]}\right)^{\mathrm{T}}\right. & \left.\boldsymbol{\chi}_{\mathrm{MS}, k}^{[m]} \odot \mathbf{a}_{\tau_{k}}^{[m]}\right) \\
& \otimes \mathbf{F}_{\mathrm{BS}, 0}^{\mathrm{H}} \boldsymbol{\chi}_{\mathrm{BS}, k}^{[m]},
\end{aligned}
$$

where $\mathbf{X}_{0}=\left[\mathbf{x}^{(0)}[0], \ldots, \mathbf{x}^{(0)}[N-1]\right]^{\mathrm{T}}$ denotes the precoded signal where $\mathbf{x}^{(0)}[n]$ is the $M_{\mathrm{MS}} \times 1$ vector of simultaneously transmitted symbols for the $n$-th subcarrier for the LOS link. The delay vector is defined as $\mathbf{a}_{\tau_{k}}^{[m]}=$ $\left[1, \ldots, e^{-j 2 \pi(N-1) \tau_{k}^{[m]} /\left(N T_{s}\right)}\right]^{\mathrm{T}}$.

The term ${ }^{5} \mathbf{F}_{\mathrm{MS}, 0}^{[m-1]}$ denotes the uplink beam selection matrix, i.e., a matrix of zeros and ones with all-zero elements in each column except the index of the corresponding beam. The beam selection matrix $\mathbf{F}_{\mathrm{MS}, 0}^{[m-1]}$ selects the corresponding beams to cover $\hat{\phi}_{0}^{[m-1]}$ with the uncertainty $\sqrt{\left[\mathbf{P}_{\boldsymbol{\psi}_{\mathrm{dl}, 0}[m-1 \mid m-1]}^{[m, 1,1}\right.}$. This angular uncertainty is obtained from the first diagonal element of the covariance estimation of $\boldsymbol{\psi}_{\mathrm{dl}, 0}^{[m]}=\left[\phi_{0}^{[m]}, \dot{\phi}_{0}^{[m]}\right]^{\mathrm{T}}$ by the EKF. In the BS, the received beam selection matrix $\mathbf{F}_{\mathrm{BS}, 0}$ is fixed and selects the corresponding beams to cover $\hat{\theta}_{0}$ with the maximum uncertainty during the observation time $T_{\mathrm{ob}}$.

\footnotetext{
${ }^{2}$ If the underlying dynamic function is unknown, a Gaussian blurring kernel centered on the previous time instant $m-1$ can be applied with a covariance corresponding to the process noise. This way, it is possible to consider timevarying rate-of-change terms.

${ }^{3}$ The NLOS paths are much weaker and susceptible to movement compared to the LOS. Consequently, they do not significantly contribute to user location and orientation tracking particularly for the outdoor scenarios [31].

${ }^{4}$ Note that the term $\tilde{h}_{0}^{[m]}$ and DL-AOA $\phi_{0}^{[m]}$ are considered as the nuisance parameters in the uplink.

${ }^{5}$ The operations $\odot$ and $\otimes$ denote the Hadamard and Kronecker products.
} 

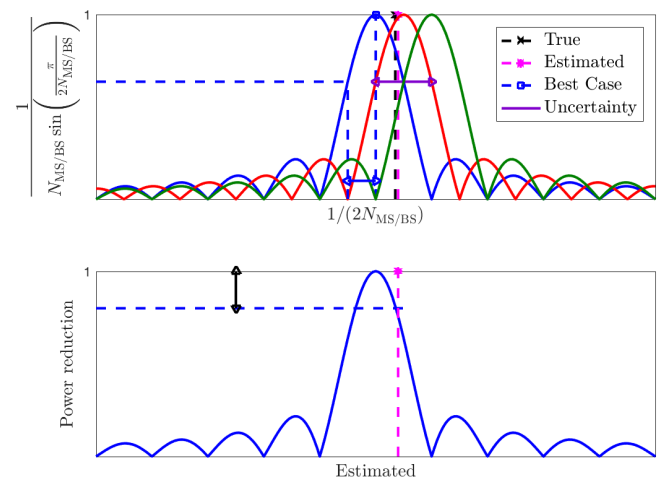

Fig. 1. The normalized magnitude of the elements of $\chi_{\mathrm{MS} / \mathrm{BS}, 0}^{[\mathrm{m}]}$ for the beam selection (top) with and (bottom) without considering the effect of uncertainty.

A similar process can be formed for tracking ${ }^{6}$ the AOA in the MS. In Fig.1, the normalized magnitude response of lens array in the BS or MS $\chi_{\mathrm{MS} / \mathrm{BS}, 0}^{[m]}$ with $N_{M S / B S}$ is shown. Ideally, the true/estimated angle should be at the pre-defined spatial direction $\left\{i / N_{M S / B S}\right\}$ for $-\left(N_{\mathrm{MS} / \mathrm{BS}}-1\right) / 2 \leq i \leq$ $\left(N_{\mathrm{MS} / \mathrm{BS}}-1\right) / 2$ to provide the maximum power. This is shown by the dashed blue line with square marker as the best case in the top plot. However, this usually does not happen as the true/estimated spatial direction might be within $1 /\left(2 N_{\mathrm{MS} / \mathrm{BS}}\right)$ from the pre-defined value. Consequently, beam selection without considering the uncertainty leads to power reduction shown in the bottom plot. This results non-robust tracking performance as will be shown in the simulations. Using the channel parameters after tracking in $\left\{\hat{\tau}_{0}^{[m]}, \hat{\theta}_{0}^{[m]}, \hat{\phi}_{0}^{[m]}\right\}$, the tracked values of position and rotation angle $\left\{\hat{\mathbf{p}}^{[m]}, \hat{\alpha}^{[m]}\right\}$ are obtained. The uncertainty of position $\mathbf{P}_{\mathbf{p}}^{[m \mid m]}$ and rotation angle $P_{\alpha}^{[m \mid m]}$ are computed as

$$
\begin{aligned}
& \mathbf{P}_{\mathbf{p}}^{[m \mid m]}=\left(\mathbf{T}_{\hat{\tau}_{0}^{[m]}, \hat{\theta}_{0}^{[m]}}^{\left[\mathbf{P}_{\boldsymbol{\psi}_{\mathrm{ul}, 0}}^{[m \mid m]}\right]_{1: 2,1: 2}^{-1}} \mathbf{T}_{\hat{\tau}_{0}^{[m]}, \hat{\theta}_{0}^{[m]}}^{\mathrm{T}}\right)^{-1}, \\
& P_{\alpha}^{[m \mid m]}=\left[\mathbf{P}_{\boldsymbol{\psi}_{\mathrm{ul}, 0}^{[m \mid m]}}^{[m \mid m]}\right]_{2,2}+\left[\mathbf{P}_{\boldsymbol{\psi}_{\mathrm{d} \mathrm{l}, 0}^{[m \mid m]}}^{[m}\right]_{1,1},
\end{aligned}
$$

where $\mathbf{T}_{\hat{\tau}_{0}^{[m]}, \hat{\theta}_{0}^{[m]}}$ denotes the conversion matrix $\mathbf{T}_{\tau_{0}, \theta_{0}}=\left[\frac{\partial \tau_{0}}{\partial \mathbf{p}}, \frac{\partial \theta_{0}}{\partial \mathbf{p}}\right]$ evaluated at $\left(\hat{\tau}_{0}^{[m]}, \hat{\theta}_{0}^{[m]}\right)$ with $\frac{\partial \tau_{0}}{\partial \mathbf{p}}=$ $\frac{1}{c}\left[\cos \left(\theta_{0}\right), \sin \left(\theta_{0}\right)\right]^{\mathrm{T}}$ and $\frac{\partial \theta_{0}}{\partial \mathbf{p}}=\frac{1}{c \tau_{0}}\left[-\sin \left(\theta_{0}\right), \cos \left(\theta_{0}\right)\right]^{\mathrm{T}}$. The term $\mathbf{P}_{\boldsymbol{\psi}_{\mathrm{ul}, 0}}^{[m \mid m]}$ denotes the uncertainty obtained by the covariance of the corresponding term $\boldsymbol{\psi}_{\mathrm{ul}, 0}^{[m]}=\left[\tau_{0}^{[m]}, \theta_{0}^{[m]}, \dot{\tau}_{0}^{[m]}, \dot{\theta}^{[m]}\right]^{\mathrm{T}}$ from the EKF. The operation $[.]_{1: 2,1: 2}^{-1}$ in (7) denotes the $2 \times 2$ upper left submatrix of the inverse of the argument.

\section{B. The Algorithm}

The heuristic beam selection and position and orientation tracking method is summarized in the Algorithm 1. The estimated channel parameters $\hat{\boldsymbol{\eta}}_{0}^{[0]}=\left[\hat{\tau}_{0}^{[0]}, \hat{\theta}_{0}^{[0]}, \hat{\phi}_{0}^{[0]}\right]^{\mathrm{T}}$ and

${ }^{6}$ For the case of asynchronous networks, rather than considering the joint AOA-AOD tracking, the tracking is performed in the UL and DL. This way, it is possible to cancel clock bias by two-way TOA estimation.

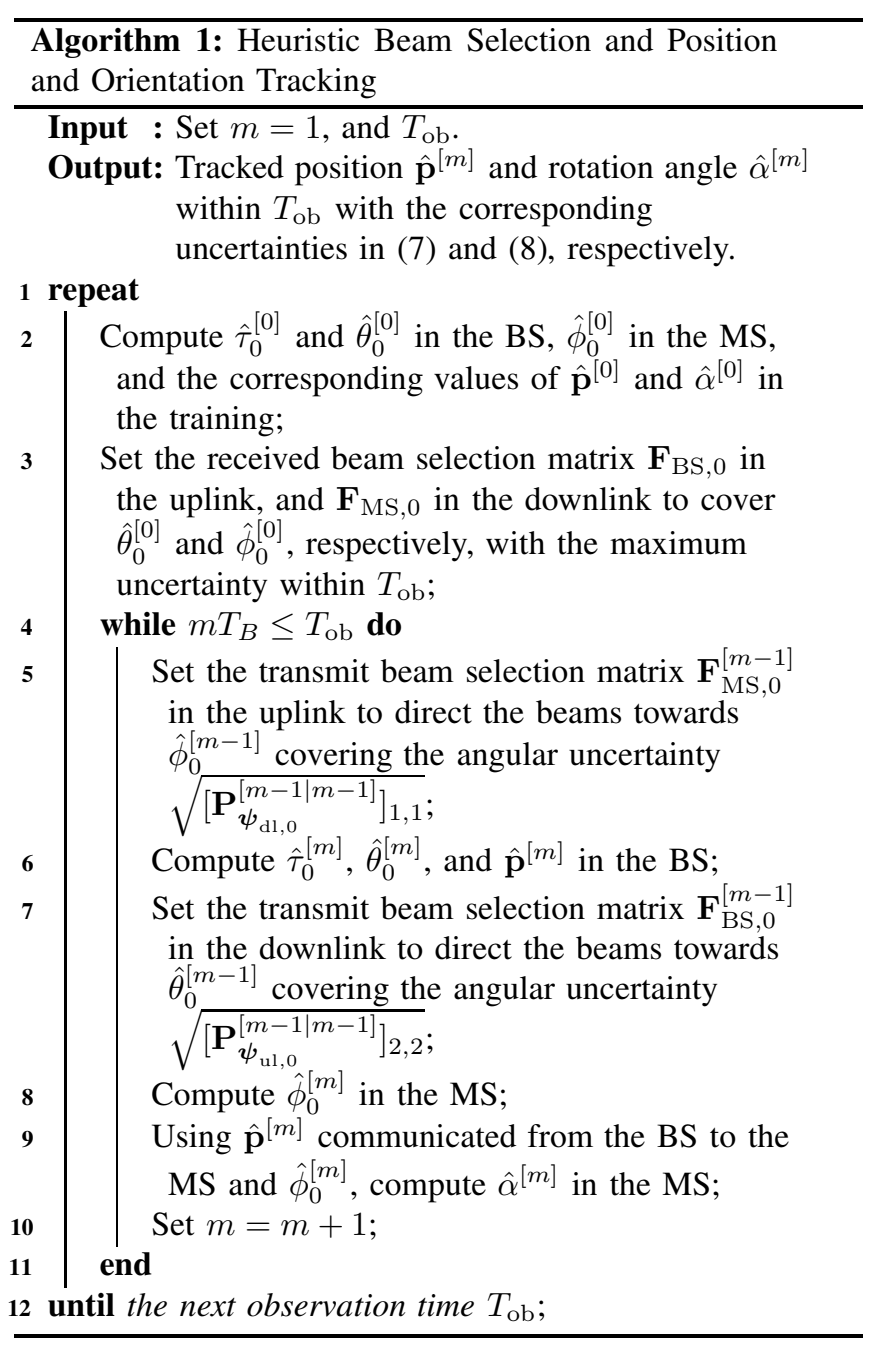

corresponding values of the position $\hat{\mathbf{p}}^{[0]}$ and rotation angle $\hat{\alpha}^{[0]}$ are obtained ${ }^{7}$ in step 2 . In step 3 , the heuristic beam selection is applied for the receiver in the uplink/downlink. In step 5, the uplink beam selection matrix $\mathbf{F}_{\mathrm{MS}}^{[m-1]}$ selects the beams directed towards the previous downlink AOA, $\hat{\phi}_{0}^{[m-1]}$, and covering the angular uncertainty $\sqrt{\left[\mathbf{P}_{\boldsymbol{\psi}_{\mathrm{d} 1,0}}^{[m-1 \mid m-1]}\right]_{1,1}}$. In step 6, the BS predicts the TOA-AOA and the location of the MS in the uplink for the current state by the analytic solution of maximum a posteriori probability (MAP) estimate:

$$
\begin{aligned}
\hat{\boldsymbol{\eta}}_{\mathrm{ul}, 0}^{[m]}=\underset{\boldsymbol{\eta}_{\mathrm{ul}, 0}^{[m]}}{\operatorname{argmin}}\left\|\check{\mathbf{y}}_{r}^{\mathrm{ul},[m]}-\mathbf{z}_{0}^{\mathrm{ul}}\left(\boldsymbol{\eta}_{\mathrm{ul}, 0}^{[m]} ; \phi_{0}^{[m]}\right)\right\|_{\mathbf{R}, 2}^{2} \\
\quad+\left\|\boldsymbol{\eta}_{\mathrm{ul}, 0}^{[m]}-\mathbf{f}_{m}\left(\hat{\boldsymbol{\eta}}_{\mathrm{ul}, 0}^{[m-1]}\right)\right\|_{\hat{\mathbf{S}}_{\mathrm{ul}, 0}^{[m]}, 2}^{2},
\end{aligned}
$$

achieved by the EKF where $\mathbf{R}$ and $\hat{\mathbf{S}}_{\mathrm{ul}, 0}^{[m]} \triangleq \hat{\mathbf{P}}_{\mathbf{f}_{m}}^{[m-1]}+\tilde{\mathbf{Q}}_{\mathrm{ul}, 0}^{[m]}$ denote the corresponding covariance of the measurement and current state, respectively. The term $\hat{\mathbf{P}}_{\mathbf{f}_{m}}^{[m-1]}$ denotes the covariance of the previous state estimate, and $\tilde{\mathbf{Q}}_{\mathrm{ul}, 0}^{[m]}$ is the

\footnotetext{
${ }^{7}$ These values can be obtained from the adopted support detection (SD)based method or simultaneous orthogonal matching pursuit (SOMP) for mm-wave lens MIMO with refinement in the uplink and downlink [10], [19], [32]. For $m>0$ they are obtained from the tracking phase.
} 
corresponding process noise covariance. The operation $\|\cdot\|_{\mathbf{M}, 2}$ stands for weighted vector norms, $\mathbf{f}_{m}($.$) is the dynamic func-$ tion that is in the form of $\mathbf{f}_{m}\left(\hat{\boldsymbol{\eta}}_{\mathrm{ul}, 0}^{[m-1]}\right)=\hat{\boldsymbol{\eta}}_{\mathrm{ul}, 0}^{[m-1]}+$ const for constant rate-of-change vector const. In step 7 , the downlink beam selection matrix $\mathbf{F}_{\mathrm{BS}}^{[m-1]}$ selects the beams directed towards the previous uplink AOA, $\hat{\theta}_{0}^{[m-1]}$, covering the angular uncertainty $\sqrt{\left[\mathbf{P}_{\boldsymbol{\psi}_{\mathrm{ul}, 0}}^{[m-1 \mid m-1]}\right]_{2,2}}$. In step 8 , the MS tracks the AOA in the downlink in a similar way as explained in step 6. In step 9, the rotation angle is obtained using the location information that is fed back to the MS and the AOA in the downlink, and the block index is updated in step 10 until $m T_{B} \leq T_{\mathrm{ob}}$. Finally, the steps 2-11 are repeated for the next observation time $T_{\mathrm{ob}}$.

\section{Simulation Results}

In this section, the performance of the proposed method for different parameters is investigated.

\section{A. Simulation Setup and Results}

We consider a scenario representative of outdoor localization based on METIS Madrid grid model [33]. We employ a ray tracing simulation tool in order to model the propagation of signals in the uplink and downlink for channel training and tracking [34]. We set ${ }^{8} f_{c}[\mathrm{GHz}]=60, B[\mathrm{MHz}]=200$, $c[\mathrm{~m} / \mathrm{ns}]=0.299792$, and $N=40$. The number of antennas in the BS and MS are set to $N_{\mathrm{BS}}=32$ and $N_{\mathrm{MS}}=32$, respectively. The received SNR in the uplink/downlink is set to $10 \mathrm{~dB}$. During the tracking, the MS moves with the velocity up to $50 \mathrm{~km} / \mathrm{h}$ suggested for outdoor vehicular mobility [33]. The angular rates for the UL-AOA and DL-AOA are up to $0.5676 \mathrm{deg} / T_{B}$ and $0.3410 \mathrm{deg} / T_{B}$, respectively. The block duration and the observation time are on the order of $T_{B}[\mathrm{~ms}] \approx 18$ and $T_{\mathrm{ob}}[\mathrm{s}] \approx 1$, respectively. The maximum angular spreads are set to $\sigma_{\theta_{0}}^{\max }[\mathrm{deg}]=\sigma_{\phi_{0}}^{\max }[\mathrm{deg}]=20$ centered around $\hat{\theta}_{0}$ and $\hat{\phi}_{0}$. During the tracking, the number of beams $M_{\mathrm{MS}}$ in the downlink and $M_{\mathrm{BS}}$ in the uplink are set to guarantee the aforementioned maximum angular supports, e.g., $M_{\mathrm{MS}}=7$ in the downlink and $M_{\mathrm{BS}}=7$ in the uplink for $N_{\mathrm{BS}}=N_{\mathrm{MS}}=32$. The power of the process noise for the continuous-time state model is set to $\mathbf{Q}_{c}=\operatorname{diag}\left\{\sigma_{\tau_{0}}^{2}, \sigma_{\theta_{0}}^{2}, \sigma_{\phi_{0}}^{2}\right\}$, and $\mathbf{Q}_{0}^{[m]}$ is obtained by numerical discretization [36]. The value of $\sigma_{\tau_{0}}$ is set to $\sigma_{\tau_{0}}[\mathrm{~ns}]=0.5$ as it only affects the tracking of the position and does not influence the rotation angle tracking. The performance of the root-mean-square error (RMSE) was assessed from 100 Monte Carlo realizations.

Fig. 2 shows the performance of the training with refinement (i.e., $m=0$ ) [19], and tracking algorithm (i.e., $m>0$ ) using heuristic beam selection with respect to the power of the process noise for the aforementioned rate of changes. The components of the standard deviation of the UL-AOA and DL-AOA process noise for the continuous-time state model $\mathbf{Q}_{c}$ are set to $\sigma_{\theta_{0}}[\mathrm{deg}]=\sigma_{\phi_{0}}[\mathrm{deg}]=\{5,12.5\}$ during the

\footnotetext{
${ }^{8}$ Although lower values of $f_{c}$, e.g., $28 \mathrm{GHz}$, are commonly used in the outdoor scenarios; however, using higher values of $f_{c}$, e.g., $60 \mathrm{GHz}$, is of great interest due to the increase in the demand of higher frequencies [35].
}
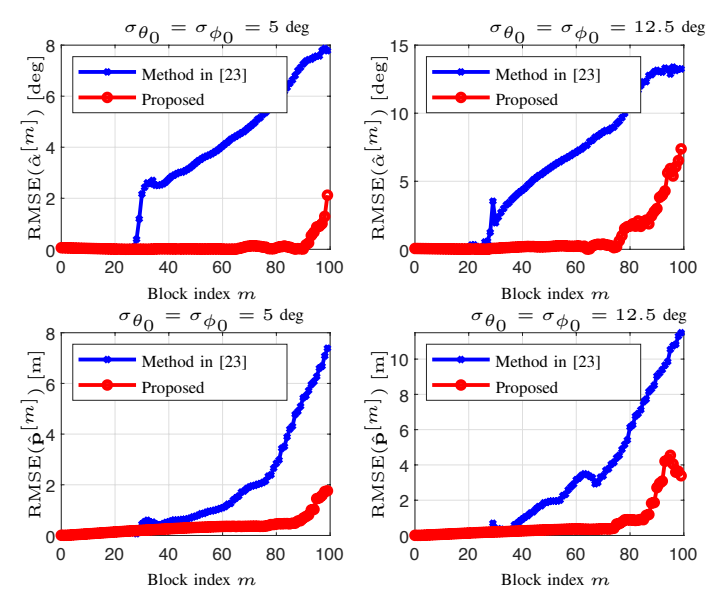

Fig. 2. The RMSE of the MS (top) rotation angle $\hat{\alpha}[m]$ and (bottom) position $\hat{\mathbf{p}}^{[m]}$ after training with refinement (block index 0) for the first 100 block indices with $N_{\mathrm{MS}}=N_{\mathrm{BS}}=32$ and $\mathrm{SNR}[\mathrm{dB}]=10$.

$T_{o b}$, i.e., on the orders of $\sigma_{\theta_{0}}[\mathrm{deg}]=\sigma_{\phi_{0}}[\mathrm{deg}] \approx\{0.1,0.2\}$ within $T_{B}$. It is observed that the RMSE of position and rotation angle gradually increases versus the block index by increasing the standard deviations of UL-AOA and DL-AOA to $\sigma_{\theta_{0}}[\mathrm{deg}]=\sigma_{\phi_{0}}[\mathrm{deg}]=12.5$ compared to the estimated values, i.e., block index zero. This is mainly due to the limited angular support of the received beam selections based on the maximum angular spreads $\sigma_{\theta_{0}}^{\max }[\mathrm{deg}]=\sigma_{\phi_{0}}^{\max }[\mathrm{deg}]=20$. On the other hand, for the standard deviations of UL-AOA and DL-AOA on the order of $\sigma_{\theta_{0}}[\mathrm{deg}]=\sigma_{\phi_{0}}[\mathrm{deg}]=5$ the RMSE of position and rotation angle are close to the values obtained from the training during $T_{o b}$, i.e., block index zero. The main reason is that the user is mostly moving within the the angular support provided by the received beam selections. After the observation time $T_{o b}$ and $\sigma_{\theta_{0}}[\mathrm{deg}]=\sigma_{\phi_{0}}[\mathrm{deg}]=5$, the user starts to move out of the angular support that leads to increasing the RMSE. For the sake of comparison, the method in [23] is adopted to the state model including the rate of change terms in (3) with lens antenna arrays. In [23], the design is not based on maximum angular spreads and EKF angular uncertainty that leads to beam misalignment and nonrobust performance. This is due to the fact that the EKF does not converge to a steady state. Consequently, the measurement cannot compensate for the increase in the angular uncertainty due to process noise by updating a selected beam with half beamwidth threshold criteria in [23]. Finally, due to tracking in the UL and the DL the proposed method requires one more transmission compared to [23].

\section{CONCLUSION}

We have studied novel solutions based on joint heuristic beam selection and position and orientation tracking in a mm-wave lens MIMO system. Through simulation studies, we have shown that the proposed method provides practical solutions for updating the location and orientation information of the user in dynamic conditions. In particular, the performance of the proposed method is close to the estimated values with the reduced complexity and pilot transmissions. 


\section{REFERENCES}

[1] P. Zhouyue and F. Khan, "An introduction to millimeter-wave mobile broadband systems," IEEE Commun. Mag., vol. 49, no. 6, pp. 101-107, Jun 2011.

[2] T. Rappaport, S. Sun, R. Mayzus, H. Zhao, Y. Azar, K. Wang, G. Wong, J. Schulz, M. Samimi, and F. Gutierrez, "Millimeter wave mobile communications for 5G cellular: It will work!" IEEE Access, vol. 1, pp. 335-349, May 2013.

[3] J. Wang, "Beam codebook based beamforming protocol for multi-Gbps millimeter-wave WPAN systems," IEEE J. Sel. Areas Commun., vol. 27, no. 8, pp. 1390-1399, Oct 2009.

[4] S. Hur, T. Kim, D. Love, J. Krogmeier, T. Thomas, and A. Ghosh, "Millimeter wave beamforming for wireless backhaul and accessin small cell networks," IEEE Trans. Commun., vol. 61, no. 10, pp. 4391-4403, Oct 2013.

[5] Y. Tsang, A. Poon, and S. Addepalli, "Coding the beams: Improving beamforming training in mmwave communication system," in Global Commun. Conf. (GLOBECOM), Kathmandu, Nepal, Dec 2011, pp. 1-6.

[6] Y. Zeng, R. Zhang, and Z. N. Chen, "Electromagnetic lens-focusing antenna enabled massive MIMO: Performance improvement and cost reduction," IEEE J. Sel. Areas Commun., vol. 32, no. 6, pp. 1194-1206, Jun 2014.

[7] Y. Zeng and R. Zhang, "Millimeter wave MIMO with lens antenna array: A new path division multiplexing paradigm," IEEE Trans. Commun. vol. 64, no. 4, pp. 1557-1571, Apr 2016.

[8] N. Behdad and A. Sayeed, "Continuous aperture phased MIMO: Basic theory and applications," in in Proc. Allerton Conference, Sep 2010, pp. 1196-1203.

[9] J. Brady, N. Behdad, and A. Sayeed, "Beamspace MIMO for millimeterwave communications: System architecture, modeling, analysis, and measurements," Trans. Antennas Propag., vol. 61, no. 7, pp. 3814-3827, Jul 2013.

[10] X. Gao, L. Dai, S. Han, C. Lin I, and X. Wang, "Reliable beamspace channel estimation for millimeter-wave massive MIMO systems with lens antenna array," IEEE Trans. Wireless Commun., vol. 16, no. 9, pp. 6010-6021, Sep 2017.

[11] L. Yang, Y. Zeng, and R. Zhang, "Channel estimation for millimeter wave MIMO communications with lens antenna arrays," IEEE Trans. Veh. Technol. DOI 10.1109/TVT.2017.2779828, Dec 2017.

[12] Y. Zeng, L. Yang, and R. Zhang, "Multi-user millimeter wave MIMO with full-dimensional lens antenna array," IEEE Transactions on Wireless Communications, vol. 17, no. 4, pp. 2800-2814, April 2018.

[13] J. Wang, J. Liu, T. Pare Jr., T. Wu, G. Bajko, and Y. Hsu, "Direction finding positioning in wireless local area networks," Mar 2017, US Patent 20170070893. [Online]. Available: http: //www.freepatentsonline.com/y2017/0070893.html

[14] P. Sanchis, J. Martinez, J. Herrera, V. Polo, J. Corral, and J. Marti, “A novel simultaneous tracking and direction of arrival estimation algorithm for beam-switched base station antennas in millimeter-wave wireless broadband access networks," in IEEE Antennas and Propagation Society International Symposium, San Antonio, TX, USA, USA, Jun 2002, pp. 594-597.

[15] H. Deng and A. Sayeed, "Mm-wave MIMO channel modeling and user localization using sparse beamspace signatures," in International Workshop on Signal Processing Advances in Wireless Communications (SPAWC), Toronto, ON, Canada, Jun 2014, pp. 130-134.

[16] M. Vari and D. Cassioli, "mmWaves RSSI indoor network localization," in International Conference on Communication (ICC) Workshop on Advances in Network Localization and Navigation, Sydney, NSW, Australia, Jun 2014, pp. 127-132.

[17] A. Guerra, F. Guidi, and D. Dardari, "Position and orientation error bound for wideband massive antenna arrays," in International Conference on Communication (ICC) Workshop on Advances in Network Localization and Navigation, London, UK, Jun 2015, pp. 853-858.

[18] A. Shahmansoori, G. Garcia, G. Destino, G. Seco-Granados, and $\mathrm{H}$. Wymeersch, " $5 \mathrm{G}$ position and orientation estimation through millimeter wave MIMO," in IEEE Global Commun. Conf. (GLOBECOM) Workshop, San Diego, CA, USA, Dec 2015, pp. 1-6.
[19] _ "Position and orientation estimation through millimeter wave MIMO in 5G systems," IEEE Trans. Wireless Commun., vol. 17, no. 3, pp. 1822-1835, Mar 2018.

[20] A. Hu, T. Lv, H. Gao, Z. Zhang, and S. Yang, "An ESPRIT-based approach for 2-D localization of incoherently distributed sources in massive MIMO systems," IEEE J. Sel. Topics Signal Process., vol. 8, no. 5, pp. 996-1011, Oct 2014.

[21] V. Savic and E. G. Larsson, "Fingerprinting-based positioning in distributed massive MIMO systems," in IEEE Vehicular Technology Conference (VTC), Boston, MA, USA, Sep 2015, pp. 1-5.

[22] N. Garcia, H. Wymeersch, E. G. Ström, and D. Slock, "Location-aided mm-wave channel estimation for vehicular communication," in Proc. IEEE International Workshop on Signal Processing Advances in Wireless Communications (SPAWC), Edinburgh, England, Jul 2016, pp. 1-5.

[23] V. Va, H. Vikalo, A. Dzul, and R. W. Heath Jr, "Beam tracking for mobile millimeter wave communication systems," in IEEE Global Conference on Signal and Information Processing (GlobalSIP), Washington, DC, USA, Dec 2016, pp. 743-747.

[24] C. Zhang, D. Guo, and P. Fan, "Tracking angles of departure and arrival in a mobile millimeter wave channel," in IEEE International Conference on Communications (ICC), Kuala Lumpur, Malaysia, May 2016, pp. 16.

[25] J. Zhao, F. Gao, W. Jia, S. Zhang, S. Jin, and H. Lin, "Angle domain hybrid precoding and channel tracking for millimeter wave massive MIMO systems," IEEE Transactions on Wireless Communications, vol. 16, no. 10, pp. 6868-6880, 2017, exported from https://app.dimensions.ai on 2019/02/04. [Online]. Available: https://app.dimensions.ai/details/publication/pub.1091035873

[26] X. Gao, L. Dai, Y. Zhang, T. Xie, X. Dai, and Z. Wang, "Fast channel tracking for terahertz beamspace massive MIMO systems," IEEE Trans. Vehicular Technology, vol. 66, no. 7, pp. 5689-5696, 2017. [Online]. Available: https://doi.org/10.1109/TVT.2016.2614994

[27] Y. Zeng and R. Zhang, "Millimeter wave MIMO with lens antenna array: A new path division multiplexing paradigm," IEEE Trans. Commun. vol. 64, no. 4, Apr. 2016.

[28] Y. Bar-Shalom, X. Li, and T. Kirubarajan, Estimation With Applications to Tracking and Navigation. Hoboken, NJ, USA: Wiley, 2001.

[29] N. Czink, X. Yin, H. Özcelik, M. Herdin, E. Bonek, and B. H. Fleury, "Cluster characteristics in a MIMO indoor propagation environment," IEEE Trans. Wireless Commun., vol. 6, no. 4, pp. 1465-1475, Apr 2007.

[30] X. Yin, T. Pedersen, N. Czink, and B. H. Fleury, "Parametric characterization and estimation of bi-azimuth dispersion path components," in Proc. of IEEE International Conf. on Signal Processing Advances in Wireless Communications (SPAWC), Cannes, France, Jul 2006, pp. 1-6.

[31] T. S. Rappaport, E. Ben-Dor, J. N. Murdock, and Y. Qiao, "38 GHz and $60 \mathrm{GHz}$ angle-dependent propagation for cellular amp; peer-to-peer wireless communications," in 2012 IEEE International Conference on Communications (ICC), June 2012, pp. 4568-4573.

[32] X. Gao, L. Dai, S. Zhou, A. M. Sayeed, and L. Hanzo, "Beamspace channel estimation for wideband millimeter-wave MIMO with lens antenna array," in IEEE International Conference on Communications (ICC), Kansas, USA, May 2018.

[33] K. e. a. Maltsev, "IEEE doc. 802.11-08/1044r0. 60 GHz WLAN Experimental Investigations," Sep 2008.

[34] N. Amiot, M. Laaraiedh, and B. Uguen, "PyLayers: An open source dynamic simulator for indoor propagation and localization," in IEEE International Conference on Communications Workshops (ICC), Budapest, Hungary, Jun 2013, pp. 84-88.

[35] H. Singh, R. Prasad, and B. Bonev, "The studies of millimeter waves at $60 \mathrm{GHz}$ in outdoor environments for IMT applications: A state of art," Wireless Personal Communications, vol. 100, no. 2, pp. 463-474, May 2018. [Online]. Available: https://doi.org/10.1007/s11277-017-5090-6

[36] M. Koivisto, M. Costa, J. Werner, K. Heiska, J. Talvitie, K. Leppänen, V. Koivunen, and M. Valkama, "Joint device positioning and clock synchronization in 5G ultra-dense networks," IEEE Trans. Wireless Commun., vol. 16, no. 5, pp. 2866-2881, May 2017. [Online]. Available: https://doi.org/10.1109/TWC.2017.2669963 